\title{
Dialectical Evolution in the Proceeds of Knowledge Development and Social Changes
}

\author{
Biplab Chattopadhyay \\ Taki Government College, Taki, North 24 Parganas, PIN - 743429, West Bengal, India
}

\begin{abstract}
Evolution is a process pertaining to the material world that shapes the course of everything, both animate and inanimate, belonging to this planet and the universe. Here, we focus on understanding as to how evolution gets going through the Hegelian dialectical paradigm and the way it influences the trails of knowledge development and social dynamic changes. In congruent endeavours, the meaning of evolution in biological systems is explored. An elaboration, with illustration, is attempted in the field of science and technology to show the optimistic influence of evolution. That, the society is prone to evolutionary changes for uplifting its state, is discussed and following of the views and concepts of natural sciences, in social dynamic changes, is emphasized.
\end{abstract}

Keywords: Evolution Cycle \& Hegelian Dialectics, Evolutionary Course of Knowledge Development, Natural Sciences Narratives of Social Dynamics, Homogeneity attribute of Stability and Freedom.

\section{INTRODUCTION}

It is a common humanistic tendency to go, quite often, by very many age old proverbial sayings describing natural phenomena. Such bent of human psyche stems from one's endeavour to read the subtleties underlying mundane nature and hence to strive to generate a sense of alert in human mind. One such saying, from a huge bunch, 'Time is invaluable', is pronounced rather clearly to indicate that worldly nature is governed by the congenital statute 'Change with elapsing Time' and it is uttered as an arousing reminder to keep up with the ever shifting disposition of nature. A pertinent question as to why changes take place at all, of which, the answer could be searched in the evolutionary route of nature. Evolution depicts that changes in nature essentially leads it to gain added homogeneity and also to acquire greater freedom in consequence.

Homogeneity connotes a specific favoured state which any entity in this material world can occupy and is characteristic of all systems exhaustively that belongs to our very own worldly nature. Any such worldly system, be it physical, chemical, biological, mathematical or similar variants, would always tend to become homogeneous following laws of nature. In similar fashion, human society, being governed by natural laws, would definitely pursue the path of homogeneity unabatedly. In case of human society the drift to homogeneity is sometimes called socialization. In any sub-variant of nature, often termed as system, homogeneity is attained through maximization of entropy (Attard, 2002) that is analogous to the number of states accessible to the system. An entropy maximum state of a system, in a sense, means rather free mixing and potentially enhanced level of interactions amongst its constituents. In allied conceptual framework, the state of socialization (homogeneity) of any magnitude in human society would mean, greater freedom for its constituent human beings in all forms of mutual interactions, uninhibited expression of views or thoughts, indiscriminate share in all social products and abundant participation in very many social processes. Here comes the natural process called evolution which makes way for a system to achieve added homogeneity or to the society the added magnitude of socialization. Acquiring the state of more homogeneity, or, to that effect, the state of being more socialized, is precisely the purpose of evolution. Every system, whatsoever, belonging to the material world, always eyes to get into the shape of a more homogeneous or socialized attire through evolutionary process.

A system pertaining to nature while takes plunge in acquiring more homogeneity feels the simultaneous urge to acquire stability too. Such stability could be achieved through evolutionary proceedings which revolve around the central idea of Darwinian natural selection (Francis, 2007). Historically Darwin's theory of evolution explains changes in biological systems. However, by now, it is an established paradigm that evolution pervades the whole of the material world of our experience and anything that evolves comes within the ambit of Darwin's theory of natural selection.

In a biological system, evolution manifests through certain dynamic phases, mostly numbered at three. In a stably continuing system, emergence of another distinctive daughter stream or that of two daughter streams negating the one parent is an eventuality and marks the germination of evolution. This emergence of two from 
one signifies materialization of a contradiction in the system. Two daughter streams, because of their unique origin, can only avail the space occupied by the parent stream. Thus, a competitive fight erupts between the two in their efforts to occupy the parental space at a specific time leading to a conflict between the two for gaining supremacy. Resolution of conflict is an essential necessity and is a binding on nature in order to uphold its own constancy because of its being limited by bounds. In settling the conflict, nature serves as the most prudent judge and does select the rather fit stream from the two contemporaries. The fittest thus survives through natural selection and acquires stability in due course attesting continuity or flow of the stream undergoing evolution. Actually, continuity of the best suited stream signifies completion of a cyclic chain in evolution from where a new cycle may get started. Therefore, everything belonging to nature always pursue the repetitive cycle of evolution so as to acquire a developed or enhanced incarnation at the edge of each completed cycle. Similar is the course of knowledge development, which follows the three definitive phases of evolution in quest for an ever elevated stature. Here, surfacing of two contrasting streams of thoughts from one, synonymise the start of contradiction which after passing through the channels of conflict and natural selection makes way for the superior or enduring stream of thought to survive and to become the existing knowledge of the aspect concerned in a rather developed form.

The contra-indicative or contradiction route of understanding evolutionary dynamics relates back to the Hegelian dialectics of $19^{\text {th }}$ century. Around the year 1808, German philosopher Georg Wilhelm Friedrich Hegel put forth a particular dialectic or method of arguments to arrive at the truth by resolving disagreements and this is broadly known as Hegelianism (Taggart and Taggart, 2011). The very basics of Hegelianism dictate that human mind cannot perceive or understand any aspect of nature unless it is cast within the frame of bipolar opposites forming a basis unit. For example, when we try to perceive 'good' we must count its opposite pole 'evil' as a benchmark and similarly understanding 'dark' would necessitate bringing in the opposite 'light' where 'dark-light' constitute the basis unity-of-opposites. This method of perceiving things or aspects pertaining to nature and its subgroup, the society, is precisely the thesis and antithesis basis of the century old Hegelian dialectics. It is fairly appealing to explore the interconnections between unstoppable evolution accounting for changes in aspectual loads of nature and the Darwin's proposition of natural selection together with the prudence of Hegelian dialectics. In the narratives of next few sections we will be devoting to exploration and explanation of the mentioned interconnections in greater detail with keen emphasis on evolutionary trail of knowledge development and social changes. We will also be focussing to investigate the areas and aspects of socio dynamic transformations where espousing of natural laws and designs are somehow vibrantly observable.

Rest of the article is organised in four further sections. In section-2, biological evolution is explored enroute the dialectical channel of contradictions and subsequent passage of the system through natural selection. Relevance and significance of the concepts of evolution in case of knowledge development is examined in section-3 and a reference is made to the knowledge upgradation in scientific and technological processes. In section-4, we try to build an understanding of the ever changing characteristic of our society from the viewpoint of evolution and in terms of concepts and laws lurking in the material worldly nature we live in. Certain summarizing comments are placed in section-5 and a bottom-line overture as epilogue is included as section- 6 in this article.

\section{BIOLOGICAL EVOLUTION AND THE DIALECTICAL CONNECT}

Evolution in a biological system is inevitable and the same is intimately connected with the essence of Hegelian dialectics. To understand the linkage of evolution with the dialectical perception of mundane aspects, let us consider the evolution of a stable species of established characteristics. It is to be emphasized that the process of evolution gets initiated through emergence of two from one pointing to the materialization of contradiction within an otherwise steady oneness. So the crux of Hegelian dialectics, as contradiction or negation cognitive, is at the helm of the process called evolution. Let us now turn back our attention to the members of a definite species which, as we all know, are exhaustively constituted by a huge but finite set of functionally coordinated organs. This is same as saying that a definite living entity is an integration of a set of finitely many organs where the more complex the species-member be, the number of functionally coordinated organs in the integrated set becomes proportionally increased (Audesirk and Audesrik, 1996). All of these organs in a living entity are undoubtedly dynamic in the sense that they suffer growth, take part in various lively syntheses and thereby keep themselves working, maintaining dynamicity for well defined purposes of life. In course of continuous and purposeful acts of organs, such as growth or synthesis, departure from expected outcome may happen because of faults created in the basic building blocks of organs, apparently termed as genes. In stricter biological terminology, one would say that while performing growth or synthesis work, organs may find occurrence of unexpected change in the genetic code and a consequent genetic mutation (Griffiths et al., 1999) in its structure and performance. Accruing mutation of a kind (or many kinds) in an aggregate of species members gets magnified and exists alongside those having organs of no genetic mutation. In course of time, mutated organs would develop a will to supersede and thus try to negate the non-mutated organ content 
members and vice versa. Ultimately, the process called natural selection takes precedence choosing the beneficial and sustainable mutations to be preserved and passed on to the next generation. Over a long timescale, beneficial mutations accumulate, magnify and result in entirely different organs from the original, thereby giving rise to a new species.

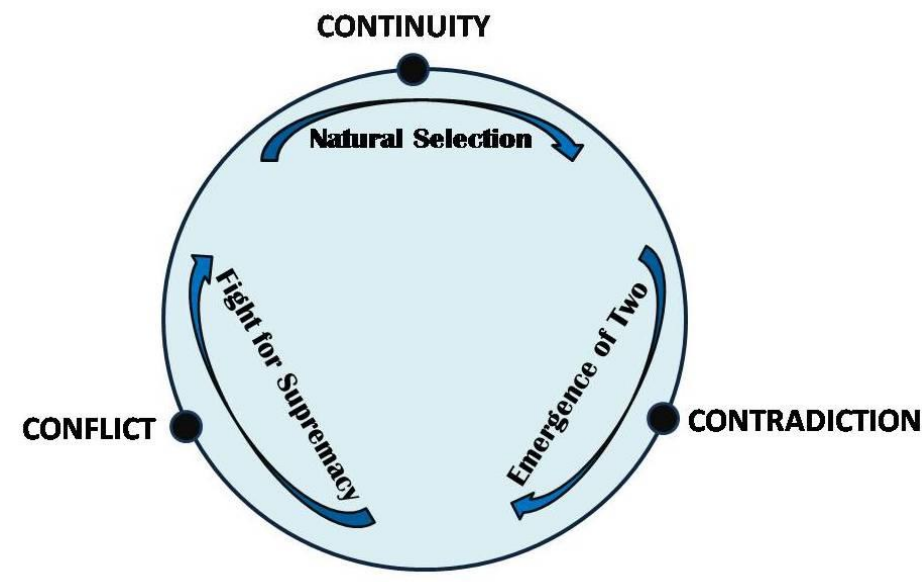

Figure-1: Illustrating the cyclic path of evolution, including its different stages, with the essence of Hegelian dialectics, that is contradiction and subsequent processes conflict and continuity being embedded within.

Biological evolution of a new species from an old one, as narrated above, is mostly the Darwin's theory that revolves around the premise of natural selection where modern era concept of mutation from molecular biology is being infused in logical terms. In this whole process of biological evolution, the essence of Hegelian dialectics, that is contradiction, plays fundamental role and denote the initiating point to the next phase of evolution. In the same spirit, two other prevalent steps of evolution, 'fight for supremacy' and 'natural selection', could be assimilated suitably with the words 'conflict' and 'continuity' respectively. Thus, the meaningful words contradiction, conflict and continuity could be identified with three prevalent phases of evolution in a cyclic path with the words signifying change-over points to different stages of evolution. An elaboration of the narrated cyclic path of evolution is imperative and is attempted below.

An organ of biological nature and defined characteristics, when gets the favour of nature and thus embark on an easy-going path, simultaneously becomes prone to random mutations in its building blocks, the genetic codes. Such mutation leads to a different organ having characteristics other than the parent one. Emergence of a characteristic new organ, disregarding the rules governing the parent organ, would, by all means, give vent to contradiction erupting in the steady stream or line. Further, in the time line, coexisting two different organs, governed by two separate bundles of rules, would try to negate each other for acquiring eminence and thus would be in serious conflict. The process of natural selection would step in here to resolve the conflict between the two and the organ having adaptable beneficial characteristics, would be permitted to sustain as well as pass on their characteristics to the descending generations illustrating continuance or continuity of beneficial mutation. The whole process of biological evolution can, thus, be picturised as three phases spread in a circle, as depicted in Figure-1, with contradiction, conflict and continuity denoting the three change-over points on the cyclic chain. Here, time is considered to flow clockwise along the cycle starting from the point of continuity, visiting phases of evolution which embed contradiction and conflict in-between and then coming to continuity again completing the specific cycle of evolution. A cyclic picture of evolutionary track signifies that evolution is unstoppable and progresses with time. Further, specific general phases of evolution are sequenced in closed chain that repeats with time in consecutive cycles with contradiction and then conflictcontinuity posing as demarcations exemplifying the importance of Hegelian dialectics in the upkeep of evolution.

\section{EVOLUTIONARY TRAIL OF KNOWLEDGE DEVELOPMENT AND ENRICHMENT OF SCIENCE}

The process of acquiring knowledge to unfold mysteries of nature, that eventually enriches human civilization, too follows the path of evolution. Existing concepts or thoughts about any natural phenomenon or any characteristic manifestation of it, as a result of ever stretching interactions, give rise to parallel set of concepts or thoughts which would definitely be in unambiguous contradiction with the established parent stream of concepts/thoughts. Each set of thoughts, flowing parallel, strives to come to prevalence and thus conflicts between them come to fore. Further, in the prospective timeline, nature itself acts as the best judge and selects 
the most logical of set of thoughts to stay on and thus get established in due course. A specific stream of thoughts or concepts, when established as appropriate (at particular space-time), becomes stable and hence continue to maintain its stability until it undergoes the next cycle of evolution. This factual espousing of evolutionary track by concepts or thoughts or knowledge and their eventual transmutation through refinement, is indicative enough that knowledge development does take place through evolutionary corridor and consequently the state of human civilization finds progressive elevation through each cycle of knowledge evolution.

Actually, development of knowledge through evolutionary trail is a necessity for progressive ascend of human civilization and this very inevitable act leads to acquisition of added insights about natural phenomena. Added insights thence generate fresh innovations inflicting renewed endeavours in all the fields of knowledge including science and technology. Thereupon, newer directions in the practice of science become observable and technological knowhow finds legitimate progress by leaps. Thence, the sequential process of evolution, demarcated by contradiction, conflict and continuity points in the close-chain cyclic track of evolution, commands significant bearing in shaping directions of contemporary science and technology by virtue of knowledge development.

The ever developing stance of science and the consequent enhancement of technological knowhow ritually follow the path of evolution. Development in science means acquiring knowledge about material abundance of nature, both animate and inanimate, in terms of plausible logical parlance and derivations. And, accumulation of scientific knowledge inherently imply enhancement of technological expertise. In general, therefore, development of knowledge is at the helm of the development of science and technology, and, here comes the playing of evolution together with the essence of Hegelian dialectics. To explore the interconnections of knowledge development with evolution along with contradiction and conflict-continuity, let us turn to a couple of specific examples.

From the time of Sir Isaac Newton, mechanical behaviour of material is understood within the ambit of Newtonian Mechanics (Muller-Kirsten, 2008) where time is considered to be absolute. Absoluteness of time claims that the time-gap between two consecutive events, as measured from different reference frames in motion relative to each other, would turn out to be the same. Counter experience to this absolute time concept had probably been there for long. However, Albert Einstein first emerged to contradict the concept of absolute time and presented logical arguments in support of his refute. The contradiction thus brought, took shape in due course into conflicting concepts about the characteristics of time-coordinate in mechanics. Resolution of the conflict was being entrusted on nature where time-perspective experimental evidences and deductive logical reasoning uphold the non-absolute character of time. Thus, the views put forth by Einstein sustained and so continued giving vent to a new branch of physics known as relativistic mechanics. However, it should be noted that Newtonian mechanics still remain in force as the initial stairs to reach the floor of relativistic mechanics which gain practical significance when relative velocity between frames is comparable to that of light.

Let us now drift to another example. During the course of scientific innovations, necessity of high magnetic field (strength) was felt at some point. Strength of magnetic field produced in an electromagnet could be increased by increasing electric current in the winding coil. However, inevitable finite resistance of winding wire, that generates heat with high current, have eventually been capping the strength of magnetic field. But, inevitability or resistance of a material, with the advent of superconductivity as evidenced by Kamerling Onnes's experiment on Mercury (Delft, 2007), is contradicted and zero resistance of material (under certain conditions) became reality. Emergence of zero resistance state conflicted the established viewpoint of heat generation by electric current and the concept of un-resisted super-current flow came to existence and persisted and continued. Here, development of knowledge through the natural evolutionary track enhanced the technological knowhow in terms of producing magnetic field of very high intensity.

\section{FOLLOWING OF NATURAL LAWS OF MATERIAL WORLD IN SOCIAL DYNAMICS}

The society we live in, is, by all means, dynamic in character and suffers changes quite evidently with the passage of time. Social processes that govern very many interactions existent among constituents of the society, too, exemplifies unhindered allegiance to evolution and consequently follows the dialectic trail of it for their own shaping as well as for realizing social changes. An evolving society, as an upshot of holistic modification of social parameters identified as social processes, apparently strive to acquire greater stability by taking plunge into a rather homogeneous state through evolution. Such adherence of the social realm to the perpetual process of evolution, manifestly imply an everlasting relevance and unscratched worth of the cyclic rolling of evolutionary progression through dialectical lead, even in the present-day social order.

Thus, by and large, it is palpable that social changes, like many other dynamic worldly phenomena, are attributed to the process called evolution. Through similar observations, as natural scientific concepts are fundamental to the built of characteristic of all terrestrial happenings, one can ascertain unambiguously that the trait of society or social processes do owe their manifestations to natural laws and their dynamic characteristics 
can be understood in terms of natural scientific concepts and views. As a matter of fact, society undergoes continuous evolutionary changes in quest for a better state of homogeneity and hence freedom for its constituents and thereby take plunge into the accessible energy minimum state. This process of social change, local or global, always remains in force, uninhibited and unabated.

Passage of the society towards more homogeneous states could be understood in terms of a space time dependent state function (or free energy functional as in physical sciences) where the entity called society always endeavours to get into the local minima of the state function. In reference to the diagram in Figure-2, let us consider that society is sitting in the minima of the state function marked A at some specified space-time. Since there exists another minima B in the proximity, lower than A, the system society would naturally tend to move to this lower minima for acquiring more homogeneity and stability by virtue of minimizing free energy. Free energy $(F)$ of any system bears the relationship (Levine, 1978)

$$
F=U-T S
$$

with its constituent internal energy $U$, temperature $T$ and entropy $S$. Quite often, a system tries to minimize its free energy through maximization of entropy. Entropy $(S)$, perceived to signify the degree of disorder of a system, is expressed as (Attard, 2002)

$$
S=k \ln \Omega
$$

where $k$ is a physical constant named after Ludwig Boltzmann and $\Omega$ stands for the number of states accessible to the system. Thus, increase in $\Omega$ would lead to enhanced entropy $S$. The meaning attached to higher $\Omega$ is that the system becomes less constrained and can be in any of the large number of states. This very concept, when translated in social terms means that constituents of the more entropic state of society would have amplified choice to remain in any of the large number of states and hence would enjoy more freedom. Further, large $\Omega$ indicates that the system constituents get evenly spread in all available states which mean a more homogeneous attire of society and enhanced liberty for its constituents. Since the state at minimum is somewhat trapped, minor fluctuations won't be able to displace it and thus the society at minimum would become more stable. The arguments above clearly establish that entropy does also indicate the level of freedom and degree of homogeneity of the constituents of a system.

Passage of the system (society) from A to B must proceed through the uphill point $\mathrm{P}$, which means an increase of the free energy. The system (society) increases its free energy (F) by virtue of increasing internal energy $(U)$ achieved by enhanced internal fluctuations. Once the system (Society) acquires free energy as that of the point $\mathrm{P}$, it rolls down to lower minimum B immediately.

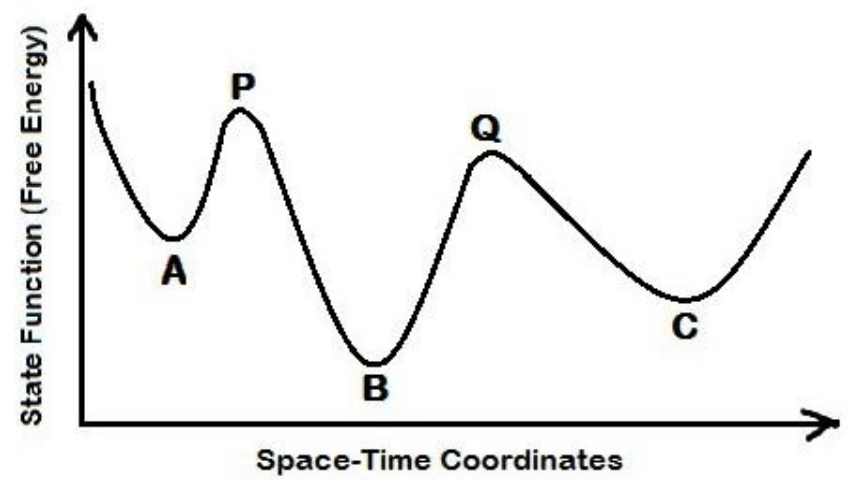

Figure-2: Diagrammatic representation of free-energy state-function with minima marked as A, B, C and uphill points (maxima) denoted as $\mathrm{P}, \mathrm{Q}$.

Narratives so far in this section emphasize the applicability of natural laws, views and concepts in case of society and thus endeavour to add knowledge to the understanding of social dynamics as well as changes occurring to the state of the society. Views, concepts and laws of nature that find application here, have got seasoned again and over through the evolutionary cycle embedding cognitive processes of contradiction, conflict and continuity and have thus got established to date with unchallenged validity. However, applicability of the natural laws, in the fashion done here for society, would probably invite modifying or fine-tuning strives through contradiction route and naturally roll on assuming the cyclic path of evolution for continuous refinement and hence a better understanding would always emerge. 


\section{SUMMARIZING COMMENTS}

In this article, our target has remained to elucidate the prototypical adherence of basic Hegelian dialectic paradigm inter alia within the evolutionary course and to emphasize that the paradigm signifies sprouting of the process called evolution. Further, we have been exploring the significance of evolution in worldly aspects like mutagenic adapt of biological entities, knowledge development and social dynamic changes. We have shown that evolutionary amends do occur in closed cycle sequence of well defined mechanisms where negation or contradiction, followed by conflict and continuity are steadfastly embedded in narrated order. Continuity in the evolutionary cycle, sitting at the loop-closing juncture, signifies steady carrying-through of the concerned change and it also gives vent to the start of a fresh cycle of evolution through anew contradictions. Bearing of evolution in the fields of Science and Technology, have been exemplified with some case studies in the allied fields. Our efforts have also been to examine as to how Hegelian philosophy of contradiction, along with recurring evolutionary cycles, lead to newer directions and innovations in the spheres of science and technology, knowledge development and in case of changing biological entities.

Our motto have furthermore been to survey the effectiveness of evolution in bringing social changes and to understand the dynamical trait of social alterations within the framework of ideas, concepts and views pertaining to natural sciences. We have endeavoured to analyze the dynamical attribute of society within the framework of natural sciences laws highlighting the way society moves from one state to the other and its tendency to attain more homogeneity in every change through evolution. Consequent upon the fact that change is an unchanging feature, one can gallantly verbalize that evolution, which inflicts changes, is an inevitable process in this world and the universe as well, and contradicting or negating the present is a must to ignite evolution, so that hope for better future remains alive.

\section{EPILOGUE: STEPS TOWARDS HOMOGENEITY}

Changes occur continually in the society which gets evolved towards the state of more homogeneity or of greater order with the objective to ward off discriminations, disparities and exploitations. The question is what proactive role the human entities should play, as potential constituent of the society, to add to the continual process of social evolution. To my mind, fighting against exploitations in its very many forms at numerous spatial locations is the task in hand which the evolving society incorporates with all spontaneity. Fights against exploitations at locations could be thought of as nucleation of small ordered blobs in the sea of disorder. With the passage of time, such blobs would grow in size and in the proximity of a relevant criticality, as correlation length gets larger, the blobs would get connected to each other. At the critical point, an infinite correlation length would make all the ordered blobs getting enjoined to a firm oneness marking a first order phase transition (Venkataraman, 1991) in the system and the whole of the society would probably be a sea of near perfect order. Such more orderly state of the society would intrinsically be of greater stability than its immediate preceding societal state and would naturally be able to afford added freedom to its constituents with enhanced parity and thereby acquiring the elevated state of homogeneity. The societal state, thus, gets moving, albeit irreversibly and in relentless progression, towards enhanced stature of homogeneity, stability and consequent emancipation. Unchanging societal traits of such compare essentially keep hopes alive for enriching human civilization for the future timeline that is unbounded.

\section{REFERENCES}

[1]. Attard P (2002) Thermodynamics and Statistical Mechanics. Elsevier Publishing.

[2]. Audesirk T and Audesrik G (1996) Biology: Life on Earth. New Jersey (USA): Prentice Hall.

[3]. Delft D van (2007) Freezing Physics. Royal Netherlands Academy of Arts and Sciences.

[4]. Francis KA (2007) Charles Darwin and the Origin of Species. London: Greenwood Press.

[5]. Griffiths AJF, Gelbart WM, Miller JH and Lewontin RC (1999) Modern Genetic Analysis. New York: W H Freeman.

[6]. Levine IN (1978) Physical Chemistry. University of Brooklyn: McGraw Hill Publishing.

[7]. Muller-Kirsten HJW (2008) Classical Mechanics and Relativity. World Scientific Publishing.

[8]. Taggart JMc and Taggart EMc (2011) Studies in the Hegelian Dialectic. Cambridge University Press.

[9]. Venkataraman G (1991) The Many Phases of Matter. INDIA: University Press.

Biplab Chattopadhyay. "Dialectic Evolution in the Proceeds of Knowledge Development and Social Changes" IOSR Journal of Humanities and Social Science (IOSR-JHSS). vol. 24 no. 06, 2019, pp. 01-06. 